Letters

\title{
How does Parkinson's disease begin? The role of compensatory mechanisms
}

\author{
Jose A. Obeso ${ }^{1}$, Maria C. Rodriguez-Oroz ${ }^{1}$, Jose L. Lanciego ${ }^{1}$ \\ and Manuel Rodriguez Diaz $^{2}$
}

\author{
${ }^{1}$ Neuroscience Centre, Clinica Universitaria and Medical School, University of Navarra, Pamplona, Spain \\ ${ }^{2}$ Department of Physiology, University of La Laguna, Tenerife, Spain
}

Bezard and colleagues [1] have to be congratulated on their studies and discussions of the mechanisms involved in compensation of striatal dopamine loss in Parkinson's disease (PD). To understand how PD begins and progresses is a major requirement in developing more definitive therapies for PD (A.E. Lang and J.A. Obeso, unpublished). Bezard et al. have conclusively shown that classically accepted dopamine-mediated mechanisms are not primarily involved in the initial compensation of striatal dopamine depletion in PD [1]. They proposed a series of functional compensatory changes within and outside the basal ganglia. We would like to discuss an alternative hypothesis for the onset of PD and the role of compensatory changes. Before that, some methodological comments are also pertinent. The Bordeaux group has established an $N$-methyl-4-phenyl1,2,3,6-tetrahydropyridine (MPTP) monkey model that provokes a full parkinsonian syndrome within three weeks, unlike PD, which develops over several years. Importantly, PD has a unilateral or asymmetrical onset whereas MPTP-intoxicated monkeys exhibited bilateral symptoms from the beginning. Both differential features of the model are relevant when analysing the onset of $\mathrm{PD}$ and initial compensatory mechanisms.

We propose that cell loss occurs asymmetrically in the substantia nigra pars compacta (SNc) in the initial, presymptomatic stage. This leads to a reduction in the dopamine-mediated innervation and functional modulation of the subthalamic nucleus (STN) (Figure 1a,b), inducing STN neuronal hyperactivity before significant dopamine striatal depletion [2,3]. At this time (in the presymptomatic state), dopamine loss in the caudal putamen is still not large enough $(<50 \%)$ to increase activity in the striatal inhibitory projection to the globus pallidus pars externa (GPe). As a result, increased STN activity exerts a powerful excitatory effect on the GPe. Excitation of the GPe leads to increased inhibition of the globus pallidus pars interna (GPi) and maintains the output of the motor circuit within normal limits. In that fashion, the 'internal' STN-GPe-GPi circuit compensates itself [4]. Increased STN activity could increase GPi firing by its direct excitatory projection [5] but this can be compensated by the still-normal 'direct' striatopallidal (i.e. to the GPi) inhibitory projection. As the

Corresponding author: Jose A. Obeso (jobeso@unav.es). neurodegenerative process progresses, striatal dopamine deficit becomes high enough (i.e. 70\%) to inhibit the GPe, diminishing its inhibitory output onto the GPi and STN (Figure 1c). At this stage, the compensatory mechanisms keeping the GPi output activity within normal limits fail and, consequently, the STN-GPi circuit becomes pathologically overactive and parkinsonian symptoms are clinically obvious. In $\mathrm{PD}$, where the changes occur slowly, excessive STN drive onto the GPi might also be compensated by way of the nigropallidal dopaminergic projection, which has been shown by fluorodopa positron emission tomography (PET) to increase during the presymptomatic phase [6]. In addition, the contralateral hemisphere probably plays an essential compensatory role, so that abnormal GPi output onto the thalamocortical projection can be compensated by the still-normally-operating basal ganglia. This is supported by PET data showing a $36 \%$ reduction in fluorodopa uptake by the hemisphere contralateral to the clinically unaffected caudal putamen in recently diagnosed patients (i.e. what appears to be normal on clinical grounds is shown to be abnormal by PET). It appears that clinical manifestation requires not only derangement of basal ganglia mechanisms in the primary affected side but also dopamine depletion in the secondarily affected side. Our interpretation does not completely fit with the physiological data of Bezard et al. [3], particularly the lack of change in firing rate of GPe neurons throughout MPTP administration. However, physiological studies in chronically intoxicated MPTP monkeys and PD patients have shown reduced neuronal firing in the GPe [7]. The major limitation of our hypothesis is basing the origin of the initial stages of $\mathrm{PD}$ on the hyperactivity of the STN and its disinhibition by a direct dopamine deficit. It could be that parallel derangement of several dopaminergic projections occurs quasi-simultaneously. Given the large cellular heterogeneity of the substantia nigra [8], early degeneration of especially vulnerable dopaminergic neurons that reach the motor putamen but that also branch, via axon collaterals, within the STN, globus pallidus and other glutamatergic nuclei (e.g. the pedunculopontine and centromedian-parafascicular nuclei), could be responsible for the hitherto unexplained increased in STN activity preceding striatal dopamine depletion. Appropriate definition of the relevance of the different compensatory mechanisms is very important for promoting new treatments for $\mathrm{PD}$. This could be feasible by using a methodology similar to the one 


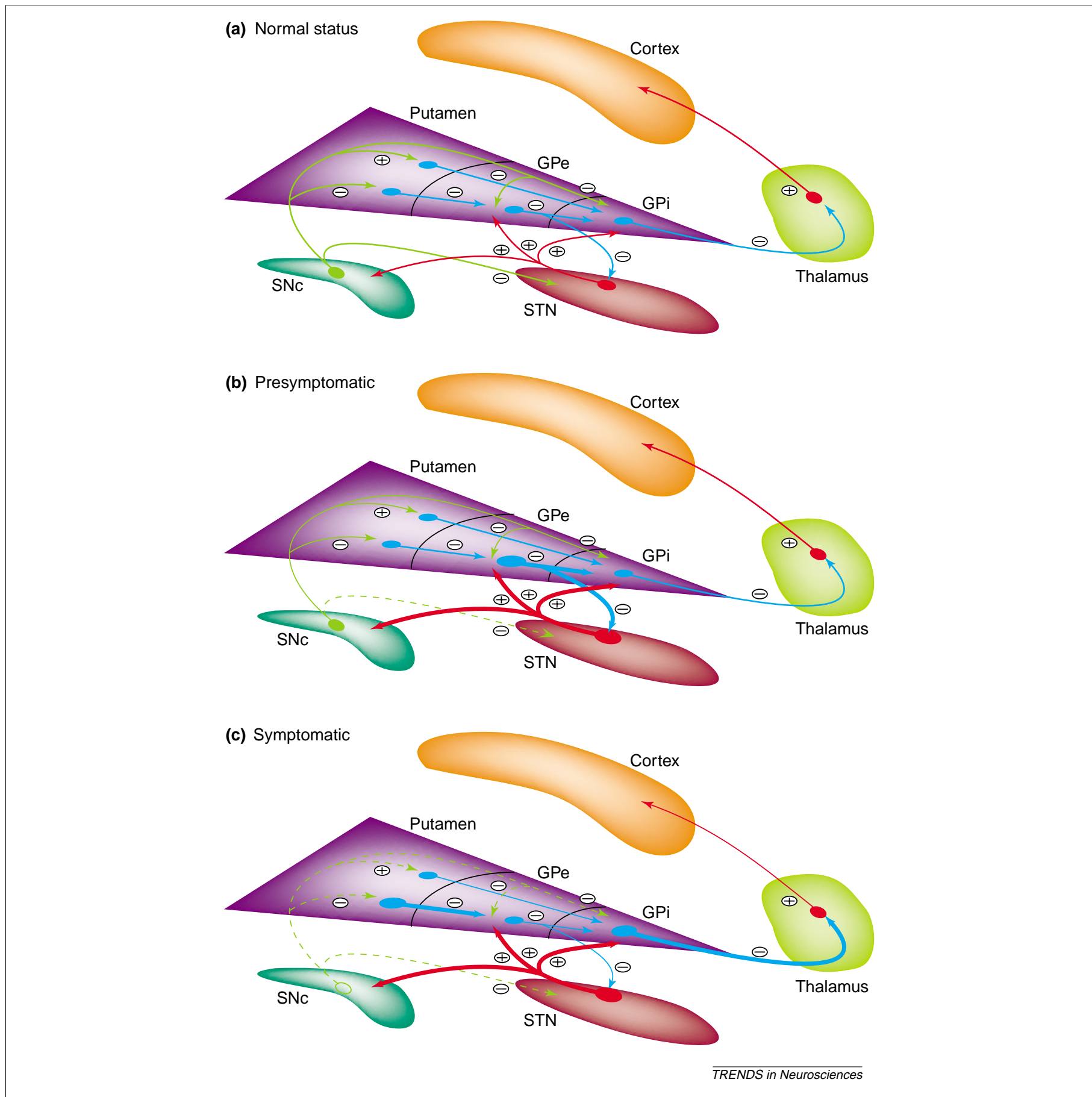

Figure 1. Modification of basal ganglia circuitry in different stages of Parkinson's disease. (a) Main basal ganglia connections in the normal state. Green arrows correspond to the dopaminergic nigrostriatal and extrastriatal projection. Blue and red arrows indicate inhibitory GABAergic efferents and excitatory glutamatergic efferents, respectively. Thickness of the arrows indicates relative functional activity. Abbreviations: GPe, globus pallidus pars externa; GPi, globus pallidus pars interna; SNc, substantia nigra pars compacta; STN, subthalamic nucleus. (b) Proposed basal ganglia modifications induced by dopamine depletion in the presymptomatic stage of Parkinson's disease. Loss of dopaminergic projections leads to hyperactivity of the STN before the onset of functional changes in the putamen. GPi output is maintained at this stage through increased inhibition from the GPe, which is excited by the STN. (c) Further dopamine loss in the putamen reaches a level that cannot be compensated for by intrastriatal mechanisms. This decreases inhibition of the 'direct' putamen-GPi projection and excessive inhibition of the GPe, which becomes hypoactive. The latter leads to further hyperactivity of the STN and GPi, accounting for the onset of parkinsonian motor features.

put forward by Bezard et al. [1,3] but that induces SNc damage much more slowly and asymmetrically.

\section{References}

1 Bezard, E. et al. (2003) Presymptomatic compensation in Parkinson's disease is not dopamine-mediated. Trends Neurosci. 26, $215-221$

2 Vila, M. et al. (2000) Evolution of changes in neuronal activity in the subthalamic nucleus of rats with unilateral lesion of the substantia nigra assessed by metabolic electrophysiological measurements. Eur. J. Neurosci. 12, 337-344

3 Bezard, E. et al. (1999) Involvement of the subthalamic nucleus in glutamatergic compensatory mechanisms. Eur. J. Neurosci. 11, $2167-2170$

4 Obeso, J.A. et al. (2000) Pathophysiology of the basal ganglia in Parkinson's disease. Trends Neurosci. 23 (Suppl 1), S8-S19

5 Whone, A.L. et al. (2003) Plasticity of the nigropallidal pathway in Parkinson's disease. Ann. Neurol. 53, 206-213

6 Shink, E. et al. (1996) The subthalamic nucleus and the external 
pallidum: two tightly interconnected structures that control the output of the basal ganglia in the monkey. Neuroscience 73, 335-357

7 Vitek, J. et al. (2000) Physiology of hypokinetic and hyperkinetic movement disorders: model for dyskinesia. Ann. Neurol. 47 (Suppl 1), S131-S140

8 Gonzalez-Hernandez, T. and Rodriguez, M. (2000) Compartmental organization and chemical profile of dopaminergic and GABAergic neurons in the substantia nigra of the rat. J. Comp. Neurol. 22 (421), $107-135$

\title{
Response to Obeso et al.: Presymptomatic compensation in Parkinson's disease is not dopamine-mediated
}

\section{Erwan Bezard ${ }^{1}$, Christian E. Gross ${ }^{1}$ and Jonathan M. Brotchie ${ }^{2}$}

\author{
${ }^{1}$ Basal Gang, Laboratoire de Neurophysiologie, CNRS UMR 5543, Université Victor Segalen, 146 rue Léo Saignat, \\ 33076 Bordeaux Cedex, France \\ ${ }^{2}$ Toronto Western Research Institute, McLaughlin Wing, Room 11-419, Toronto Western Hospital, 399 Bathurst Street, \\ Toronto, ON M5T 2S8, Canada
}

Obeso and colleagues address several issues [1], two of which are particularly relevant. The first, and most crucial, issue with regard to the findings reported in our initial review [2] relates to the non-human-primate model of Parkinson's disease (PD) we have developed to investigate compensatory mechanisms. The criticism is essentially that, in our model, chronic administration of low-dose $\quad N$-methyl-4-phenyl-1,2,3,6-tetrahydropyridine (MPTP) produces uniform striatal dopaminergic denervation [3]. This is also reported for acute intoxication with high MPTP doses in both monkey [4] and human [5]. We acknowledge that, although our model makes significant advances over previous ones in terms of showing progression, in this respect it diverges from the clinical situation, in which subdivisions within the human substantia nigra pars compacta (SNc) [6] are differentially affected by progression of the disease $[7,8]$. The difference might reflect differences in the site of the initial trigger for degeneration in the model versus PD (i.e. striatal versus $\mathrm{SNc}$ ). It has been suggested that in PD there is a mesencephalic origin for the pathological process [9], but that different sensitivity of different localities within SNc and ventral tegmental area (VTA) that have different projection zones leads to a gradient in dopamine depletion, with a higher loss in dorsal and caudal parts of the putamen than in the caudate nucleus. However, because the active metabolite of MPTP is taken up by the dopamine transporter which is distributed homogeneously across the striatum, MPTP leads to a more uniform striatal denervation [10]. Further understanding of mechanism of neuronal death in PD will be required before developing a new model that mimics the details of the pattern of degeneration. In any case, the real issue is whether the lack of a PD-like pattern of degeneration has an impact on the interpretation of our findings with respect to identification of novel com-

Corresponding author: Erwan Bezard (erwan.bezard@umr5543.u-bordeaux2.fr). pensatory mechanisms. We believe not. Compensation will occur as dopamine is lost in the striatum and the effects of that loss are propagated through the circuit. In the case of this model, such compensation will not be subject to topographical variation but might well be uniformly spread across the circuit. The uniformity of the depletion could actually work in favour of the model, making it possible to define presymptomatic compensatory changes clearly.

The above notwithstanding, Obeso et al. raise a very interesting point relating to the potential functional consequences of the asymmetric loss of dopamine in PD and how lack of such asymmetry might have an impact on the interpretation of our data. They propose that in PD, asymmetric degeneration could lead to loss of dopamine input to the STN before significant striatal loss and that this leads to a compensatory STN overactivity that drives the remaining dopaminergic cells to release more dopamine. However, although conceptually appealing, the hypothesis is not supported by experimental evidence. In fact, the STN has been shown to receive dopaminergic fibers $[11,12]$ that originate from throughout the SNc-VTA complex [13]. This projection is actively spared in parkinsonian monkeys [14-17]. It is, thus, hard to reconcile these findings with an early loss of dopamine-mediated control of STN. Moreover, of great interest is the recent finding that dopamine actually potentiates burst-firing in STN neurons through the activation of $D_{5}$ receptors [18]. A loss of dopamine in the STN would thus not necessarily lead to an increased activity. In fact, we would propose that, because dopamine input to the STN is relatively spared, because dopamine provokes burst-firing in STN neurons and because burst-firing is a key feature of pathological STN activity in both MPTP monkeys [19] and PD patients [20], it is unlikely that (i) a specific loss of STN dopamine input occurs in presymptomatic PD, and (ii) this loss is compensatory. By contrast, the relative sparing of the pathway could contribute to symptom 